\title{
NEW RESULTS FOR PHOTOIONIZATION AND RECOMBINATION OF ASTROPHYSICALLY ABUNDANT ATOMS AND IONS: THE CARBON SEQUENCE
}

\author{
Sultana N. Nahar and Anil K. Pradhan \\ Department of Astronomy, Ohio State University, Colombus, OH 43210 \\ Received 1992 January 27; accepted 1992 April 8
}

\begin{abstract}
Photoionization cross sections and electron-ion recombination rate coefficients are presented for carbon-like ions obtained using methods developed for the Opacity Project. General features of the new data are discussed, and their astrophysical relevance is pointed out. The illustrative results for photoionization include extensive autoionization resonance structures and partial photoionization into specific states of the residual ion. It is shown that the excited state photoionization cross sections are not, in general, described by hydrogenic behavior and often contain strong resonances due to photoexcitation corresponding to dipole transitions in the core ion; these are referred to as photoexcitation-of-core resonances that attenuate the background cross section over much larger energy ranges than the Rydberg type of resonances. The effective photoionization cross sections are thus considerably enhanced, for excited bound states, relative to the hydrogenic form. A new method is described for the calculation of total recombination rate coefficients that accounts for both the radiative and the dielectronic recombination processes in an ab initio manner. The recombination calculations are carried out in the close coupling approximation using the Milne relation with detailed photoionization cross sections for large numbers of excited states of each atom or ion, and employing a precise theory of dielectronic recombination given by Bell $\&$ Seaton. The present recombination rate coefficients are compared with earlier works on radiative and dielectronic recombinations treated separately and significant differences are noted. Recombination rate coefficients are presented for $\mathrm{C}$ I, $\mathrm{N}$ II, $\mathrm{O}$ III, F IV, and $\mathrm{Ne} v$ at a wide range of temperatures for astrophysical applications.
\end{abstract}

Subject headings: atomic data - atomic processes

\section{INTRODUCTION}

A large amount of accurate radiative data, oscillator strengths, and photoionization cross sections has been calculated by the Opacity Project for nearly all astrophysically abundant elements with $Z=1-14,16,18,20,26$ in all ionization stages (Seaton 1987; Pradhan 1987). In this report we focus on the astrophysical implications of the new data on photoionization cross sections and total (electron + ion) recombination rate coefficients. Some of the significant features have been discussed in detail by Yu \& Seaton (1987) and Nahar \& Pradhan (1991, hereafter NP1). The Opacity Project work is based on the $R$-matrix method from atomic collision theory that is capable of precise and large-scale treatment of a variety of atomic processes. A number of theoretical and computational developments have been carried out to enable extensive and accurate calculation of radiative and collisional data. The methods are described by Berrington et al. (1987). The Opacity Project is an international collaboration with groups of researchers in the UK, the US, Germany, France, and Venezuela.

By and large, astrophysical models rely on atomic data calculated in relatively simple approximations that often do not account for important atomic effects, such as autoionization resonances and interchannel coupling between atomic states, and may entail uncertainties of several factors relative to cases where the two effects are neglected. Also, the atomic data for excited states of atoms and ions are not usually available, and consequently the photoionization models for active galactic nuclei (AGNs), quasars, $H$ II regions, etc., and non-LTE stellar models employ hydrogenic data for these states. The models depend critically on continuum radiative processes as ioniza- tion balance and spectral formation are driven by central radiation sources. The convolution of the radiation field source function with the photoionization cross sections on the one hand, and the effective electron-ion recombinations on the other, should both be influenced by the level of detail, extent, and accuracy of the new work based on the Opacity Project (hereafter OP) work.

Basically, the OP data for photoionization differs from earlier calculations in the following respects.

1. A large number of excited bound states are considered explicitly and photoionization cross sections are available for these states for the first time. Furthermore, the photoionization of the excited states often departs considerably from hydrogenic behavior normally assumed in present astrophysical models.

2. Autoionization structures have been delineated in detail; these affect the photoionization cross sections over extended energy ranges, in particular in the important near-threshold region.

3. Partial cross sections may be obtained for photoionization into excited states of the residual ion; these are of importance in non-LTE stellar models where the population rates of several excited states, following photoionization, need to be determined precisely (particularly the metastables and the lowest states connected to the ground state by dipole allowed transitions).

4. Total recombination rate coefficients may be obtained accurately from a new method for electron + ion recombination (Nahar \& Pradhan 1992a, hereafter NP2) based on the detailed OP photoionization cross sections and the precise theory of dielectronic recombination developed by Bell \& Seaton (1986). The OP photoionization data consist of cross sections for bound states up to $n \leq 10$, which includes autoion- 
izing resonances in an ab initio manner. The application of the Milne relation, with the assumption that the resonances decay predominantly into the continuum, yields total effective recombination rate coefficient, $\alpha_{R}$, that includes both radiative recombination (RR) and dielectronic recombination (DR). These have usually been considered separately in previous works. In the high-energy region with $n>10$, below the thresholds of convergence for the Rydberg series of autoionizing states, DR dominates RR and the recombination rate is supplemented by the DR contribution according to the Bell \& Seaton theory of dielectronic recombination.

In the following sections we discuss these points in some detail with selected new results of photoionization of carbonlike ions.

\section{PHOTOIONIZATION}

A number of photoionization results from $O P$ have been presented elsewhere (e.g., Yu \& Seaton 1987; NP1). Here we shall present selected examples of the features in the OP photoionization data that involve new atomic physics incorporated in current astrophysical models.

\subsection{Ground and Excited States}

Usually one considers only the ground state photoionization cross section in detail; the excited states are treated in the hydrogenic approximation (e.g., Aldrovandi \& Pequignot 1973). The OP calculations include photoionization from a large number of excited bound states; for example, 159 bound states of C I (discussed later). The number of excited states increases with the degree of ionization along an isoelectronic sequence; nearly 800 states are considered for C-like iron. The data reveal that the photoionization of most of the excited states deviates considerably from the hydrogenic behavior not only for the low-lying states but also for the highly excited Rydberg states (see next section). Figure 1 shows the photoionization cross sections of the ground and two excited states, $2 s 2 p^{3}\left({ }^{3} D^{\circ}\right)$ and $2 s^{2} 2 p 3 d\left({ }^{3} F^{\circ}\right)$ of $\mathrm{C}$. As may be seen the cross section for the excited state is quite different from the $\omega^{-3}$ behavior characteristic of hydrogenic photoionization.

The important consideration for photoionization models then is that a number of excited states should be considered explicitly. It is also evident that recombination to the excited states will play a role in the usual equation for photoionization equilibrium (Osterbrock 1989) which we write as

$$
\begin{aligned}
\sum_{i} \int_{v}^{\infty} \frac{4 \pi J_{v}}{h v} N\left(X_{i}^{n+}\right) \sigma_{\mathrm{Pl}}\left(v, X_{i}^{n+}\right) d v & \\
& =\sum_{j} N_{e} N\left(X^{n+1}\right) \alpha_{R}\left(X_{j}^{n+} ; T\right),
\end{aligned}
$$

where $\sigma_{\mathrm{PI}}$ is the photoionization cross section.

Implicit in equation (1) is the assumption that the sums on either side may involve different states of the ionizing and the recombining ions; the sum on the right-hand side extends over the many excited states whose recombination rate coefficients must be obtained from detailed photoionization calculations.

\subsection{Autoionization Structures}

The close coupling approximation employed in the OP photoionization calculations allows for the effect of autoionization of the (electron + ion) states lying above the ionization threshold. In general there are several Rydberg series of resonances belonging to the excited thresholds of the residual ion, in

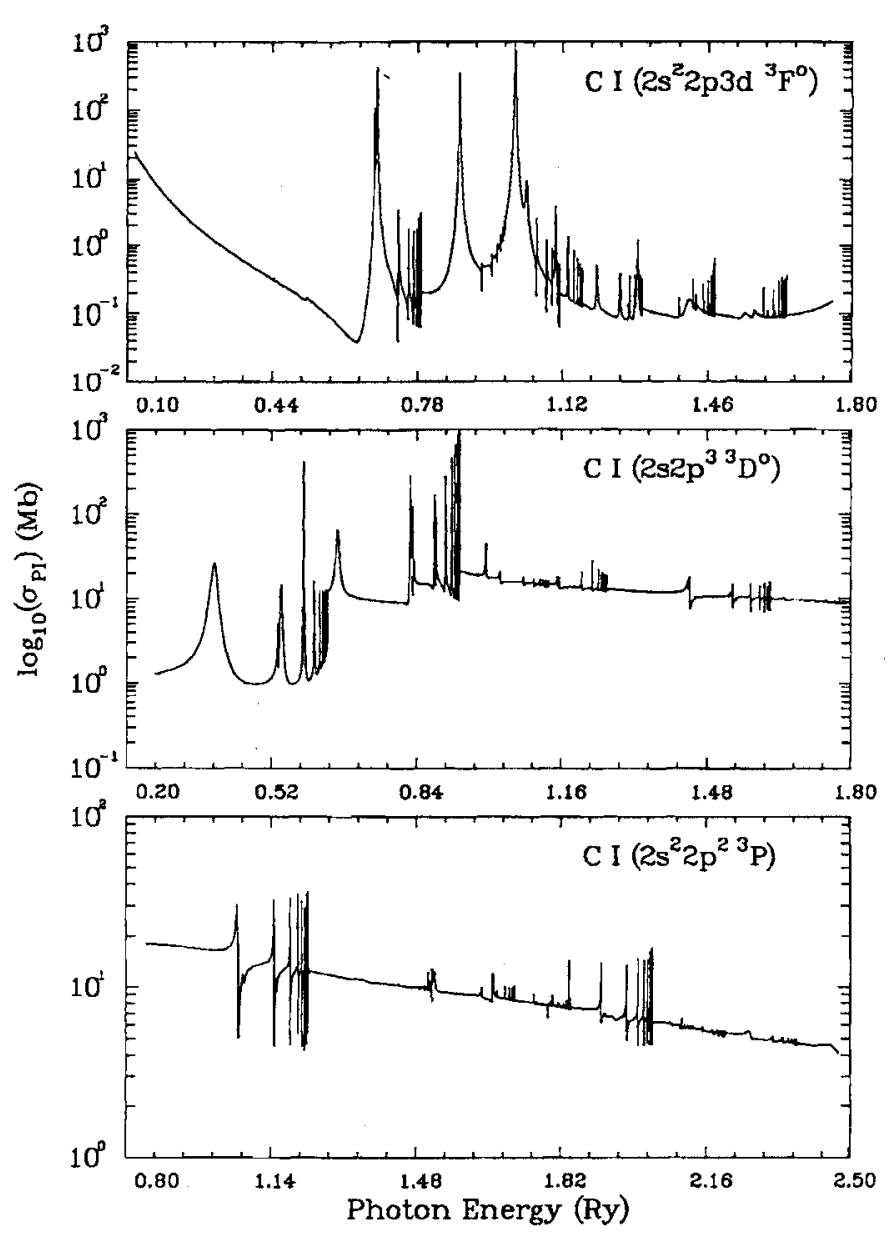

FIG. 1. Photoionization cross sections of the ground state $2 s^{2} 2 p^{2}\left({ }^{3} P\right)$ and the excited states $2 s 2 p^{3}\left({ }^{3} D^{o}\right), 2 s^{2} 2 p 3 d\left({ }^{3} F^{o}\right)$ of $C_{1}$.

the photoionization cross sections. The resonance structures are delineated using two different meshes (NP1): (1) a fine mesh of constant energy for the near threshold region and (2) a constant quantum defect mesh up to the highest threshold of the residual ion. For the quantum defect mesh, there are 100 points for each interval of 1.0 of effective quantum number $v$, going up to $v=10.0$ of Rydberg series relative to the excited ionization thresholds of the residual ion. Resonances with $v>10$ are averaged using the Gailitis averaging procedure (e.g., Yu \& Seaton 1987 and NP1).

One particular type of resonances that is found to be a common feature in excited state photoionization cross sections is the so-called photoexcitation of core (PEC) resonances that occur at photon energies corresponding to the energy differences between states of the residual ion related by dipole allowed transitions. In this process the electron recombines into a Rydberg autoionizing state, exciting the ion core, but remains essentially a "spectator" while the ion core radiates back to the ground state and the electron decays into the continuum (dielectronic recombination takes place if the electron remains bound). Figure 2 shows the photoionization cross sections of the Rydberg series of excited bound states $2 s^{2} 2 p\left({ }^{2} p^{\circ}\right) n f\left({ }^{3} F\right)$ of $C$ I that exhibit the PEC resonance features corresponding to the four dipole allowed transitions in the 


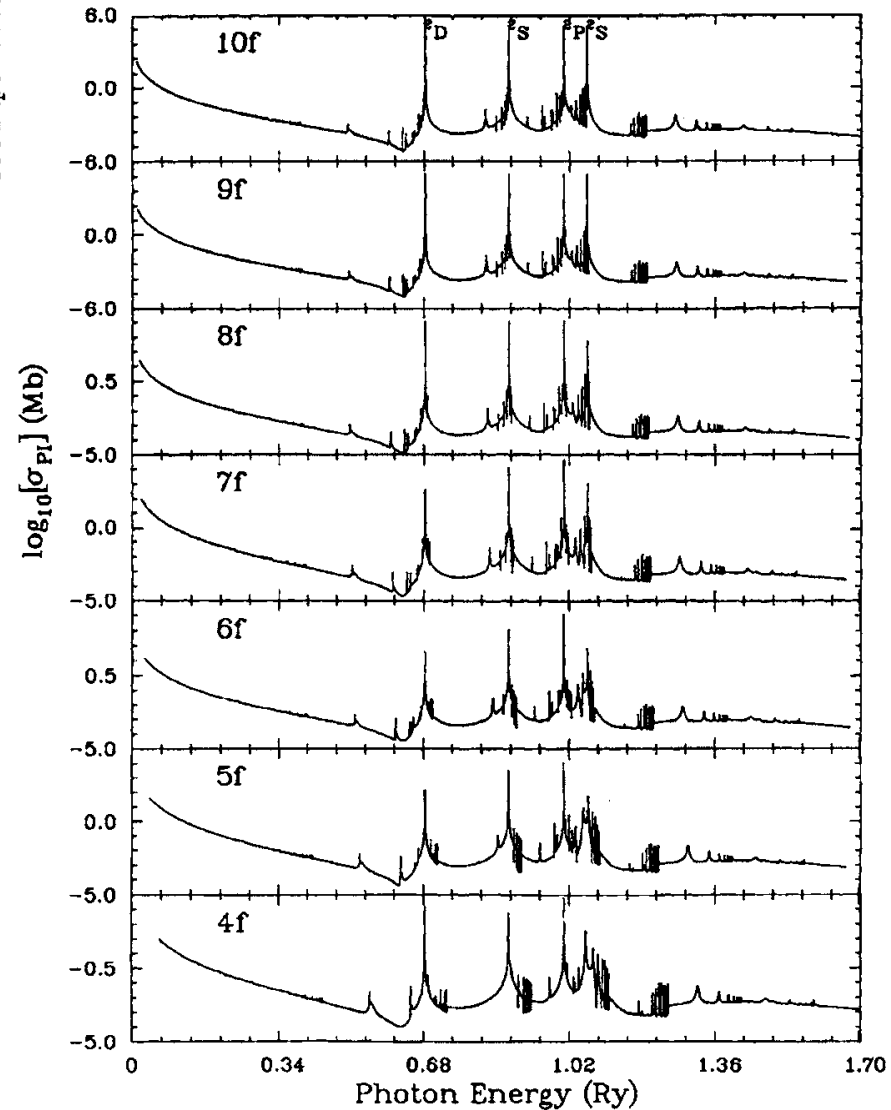

Fig. 2--Photoionization of the Rydberg series of bound states of $\mathrm{Cr}$ : $\left.2 s^{2} 2 p^{2} P^{\circ}\right) n f\left({ }^{3} F\right), 4 \leq n \leq 10$. The PEC resonances are illustrated corresponding to the core transitions $\left.\left.2 s^{2} 2 p^{2} P^{0}\right) \rightarrow 2 s 2 p^{2}\left({ }^{2} D,{ }^{2} S,{ }^{2} P\right), 2 s^{2} 3 s^{2} S\right)$.

residual ion $\mathrm{C} \mathrm{n}$ :

$$
2 s^{2} 2 p\left({ }^{2} P^{o}\right) \rightarrow 2 s 2 p^{2}\left({ }^{2} D,{ }^{2} S,{ }^{2} P\right), 2 s^{2} 3 s\left({ }^{2} S\right) .
$$

The PEC resonances attenuate the photoionization cross sections over broad energy ranges, and many enhance the cross sections by orders of magnitude over background values, which one might otherwise expect to be hydrogenic for Rydberg states of the type $\left({ }^{2} P^{0}\right) n f$.

\subsection{Partial Photoionization Cross Sections}

Under conditions of non-LTE astrophysical models require the determination of level populations due to photoionization in excited states of ions, in addition to the ground state. The close coupling approximation for photoionization enables the calculation of partial cross sections into states of the residual ion coupled in the eigenfunction expansion. Typically we include all states dominated by the ground complex of configurations; for example, the states included in eigenfunction expansion for $\mathrm{O}$ iv are $1 s^{2} 2 s^{2} 2 p\left({ }^{2} P^{\circ}\right), 1 s^{2} 2 s 2 p^{2}\left({ }^{4} P,{ }^{2} D,{ }^{2} S,{ }^{2} P\right)$, and $2 p^{3}\left({ }^{4} S^{0},{ }^{2} D^{o},{ }^{2} P^{0}\right)$. Partial cross sections may therefore be obtained for photoionization of $\mathrm{O}$ III into all eight states of $O$ IV in the coupled representation. In Figure 3 we present partial and the total photoionization cross sections for the ground state ${ }^{3} P$ of $O$ III.

It might be noticed from Figure 3 that for some of the excited states into which the residual ion is formed, following photoionization, the near-threshold resonances may enhance

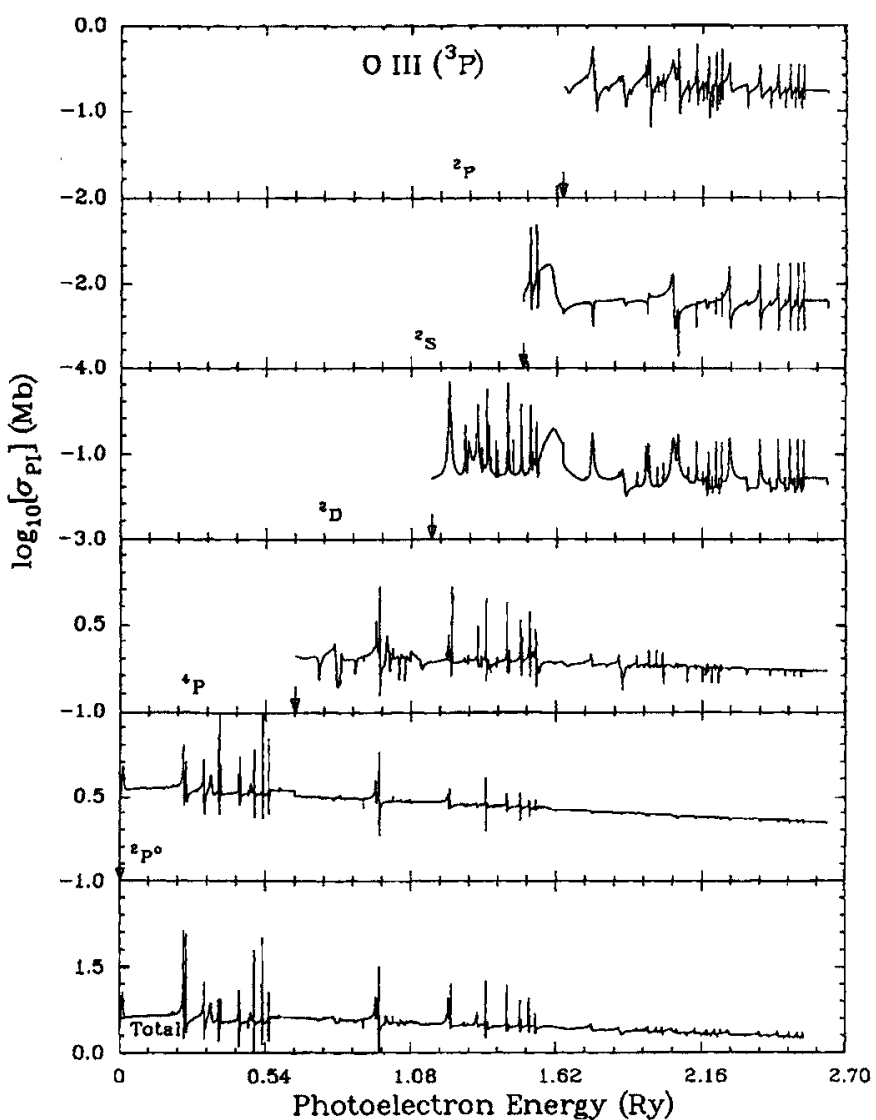

FiG. 3.-Total (bottom panel) and partial (upper panels) photoionization cross sections for the ground state $2 s^{2} 2 p^{2}\left({ }^{3} P\right)$ of $O \mathrm{IIl}$, with the residual ion $O$ iv in states ${ }^{2} P^{\circ},{ }^{4} P,{ }^{2} D,{ }^{2} S$, and ${ }^{2} P$.

the population rate; for example, in the ${ }^{2} D$ partial cross section.

More detailed results for the partial and total photoionization cross sections of the ground states of the carbon sequence ions $\mathrm{C}_{\text {I }}, \mathrm{N}$ II, $\mathrm{O}$ III, F IV, Ne v, Na VI, Mg VII, Al VIII, SI IX, $\mathrm{S} \mathrm{xI}, \mathrm{Ar} \mathrm{XII}, \mathrm{Ca} \mathrm{XV}$, and $\mathrm{Fe}$ XVII are given by Nahar \& Pradhan (NP1 1991; NP3 1992b).

\section{ELECTRON-ION RECOMBINATION}

The total electron-ion recombination process is usually viewed as two separate processes of radiative and dielectronic recombinations, owing to the fact that the effect of autoionization is not considered in the photoionization itself. The OP work, however, includes the resonances and therefore an application of the Milne relation between photoionization and recombination yields the effective, combined recombination rate coefficient (Osterbrock 1989)

$$
\alpha_{R}(T)=\frac{g_{i}}{g_{+}} \frac{2}{\left(2 \pi m^{3}\right)^{1 / 2} c^{2}} \frac{e^{((f / k T)}}{(k T)^{3 / 2}} \int_{0}^{\infty} E^{2} \sigma_{\mathrm{Pr}}(E) e^{(-E / k T)} d E .
$$

In the low-temperature range the rate may be considerably enhanced due to near-threshold resonances in $\sigma_{\mathrm{pr}}$ (Nussbaumer \& Storey 1983). Particular care is needed to ensure that the near-threshold resonances are included and their positions accurately determined. In the present work, and in NP1 and NP3, resonances with principal quantum number 
$n \leq 10$ are obtained using two different meshes to enable a fine resolution of resonances profiles. Further, to obtain precise position of the resonances we employ spectroscopically observed energies for the states of the residual ion, relative to which the resonance structures are delineated. It is impractical to consider increasingly narrower resonance profiles with $n>10$ where the background cross section is insignificant. However, there are an infinite number of resonances, in each cross section, corresponding to the Rydberg series of autoionizing states converging on to the various thresholds of the core ion. In the region below these thresholds the recombination process is dominated by $\mathrm{DR}$, and most of the DR contribution arises from these Rydberg resonances with high $n$ values, generally $n \gg 10$. Bell \& Seaton (1986) have presented a precise, ab initio theory of DR that has not heretofore been employed in the calculation of total recombination rates. NP2 have developed a computational formalism that combines both the OP photoionization data, using the Milne relation as described above and in NP1, and the Bell \& Seaton treatment of DR to obtain total electron + ion recombination rate coefficients. As the NP2 formalism is based on the close coupling approximation (using the $R$-matrix method), it is fully consistent with the close coupling calculations for electron-ion scattering and photoionization. In fact, the same eigenfunction expansion for the residual ion states may be used in all three types of calculations, i.e., for photoionization, scattering, and recombination.

The new method for DR enables the calculation of detailed and resonance averaged DR collision strengths, $\Omega(D R)$, as discussed in NP2. The predominant contribution to DR comes from the resonances and other low-lying dipole allowed tran- sitions within the ion core. The DR collision strengths, $\Omega(D R)$, are peaked at the thresholds of excitation of the allowed transitions. Figure 4 illustrates $\langle\Omega(D R)\rangle$ of the $e+N$ III $\rightarrow \mathrm{N}$ II system. The computation of $\langle\Omega(D R)\rangle$ is carried out from an energy corresponding to effective quantum number $v=10.0$ which corresponds to $n=10$ (marked by arrows in Fig. 4). This choice is made since the detailed photoionization cross sections have been calculated for all bound states of $\mathrm{N}$ II with $n \leq 10$. It is assumed that the recombining electron is a spectator electron, with $n>10$, that does not play an active role in the radiation process and the principal quantum number remains unchanged following radiative decay of the core. The magnitude of the DR collision strengths at the peak values is exactly equal to the collision strength for the inelastic electron impact excitation (EIE) at the corresponding threshold, e.g., $\Omega\left(\mathrm{DR} ;{ }^{2} D\right)=\Omega\left(\mathrm{EIE} ;{ }^{2} P^{\circ}-^{2} D\right)$. [ $\Omega(\mathrm{EIE})$ are represented by filled circles in Fig. 4.]

As the OP data consist of photoionization of a large number of excited states of each atom or ion, typically a few hundred bound states, recombination rate coefficients may be obtained for all of those states individually, as well as the total.

\subsection{Total Recombination Rate Coefficients for Carbon-like Ions}

In the present work we present total recombination rate coefficients for boron-like target ions to form carbon-like $\mathrm{C}$, $\mathrm{N} \mathrm{II,} \mathrm{O} \mathrm{m,} \mathrm{F} \mathrm{IV,} \mathrm{and} \mathrm{Ne} \mathrm{V}$. In order to illustrate the nature of the present work in relation to the currently available data we present rate coefficients for the first three ions in Figures $5 a-5 c$
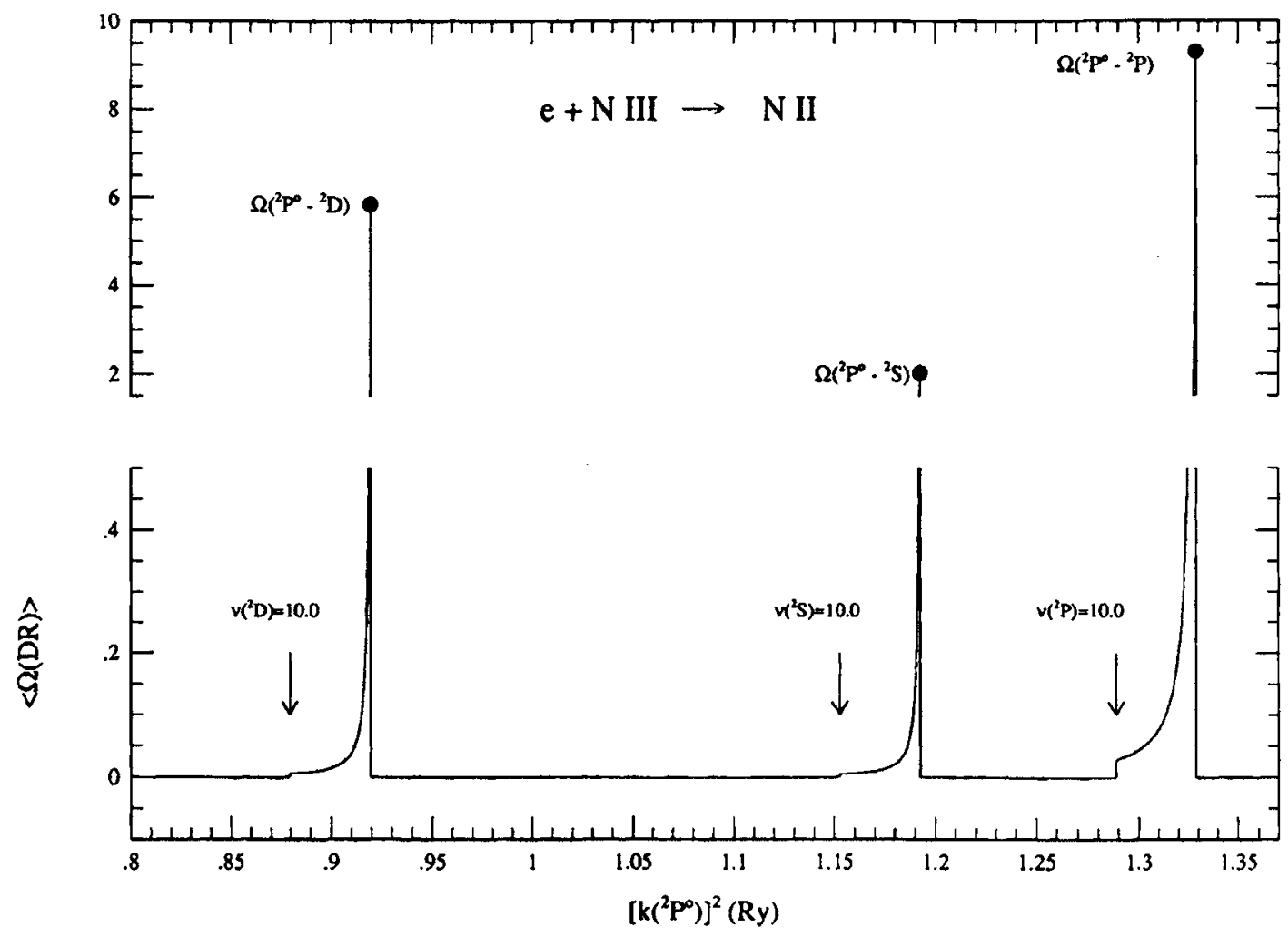

FIG. 4.-Averaged collision strength for dielectronic recombination, $\Omega$ (DR), of $e+N$ uI $\rightarrow \mathrm{N}$ II (solid curve). The filled circles represent collision strength for electron impact excitations, $\Omega$ (EIE), for transitions ${ }^{2} P^{\circ} \rightarrow\left({ }^{2} D,{ }^{2} S,{ }^{2} P\right.$ ). 


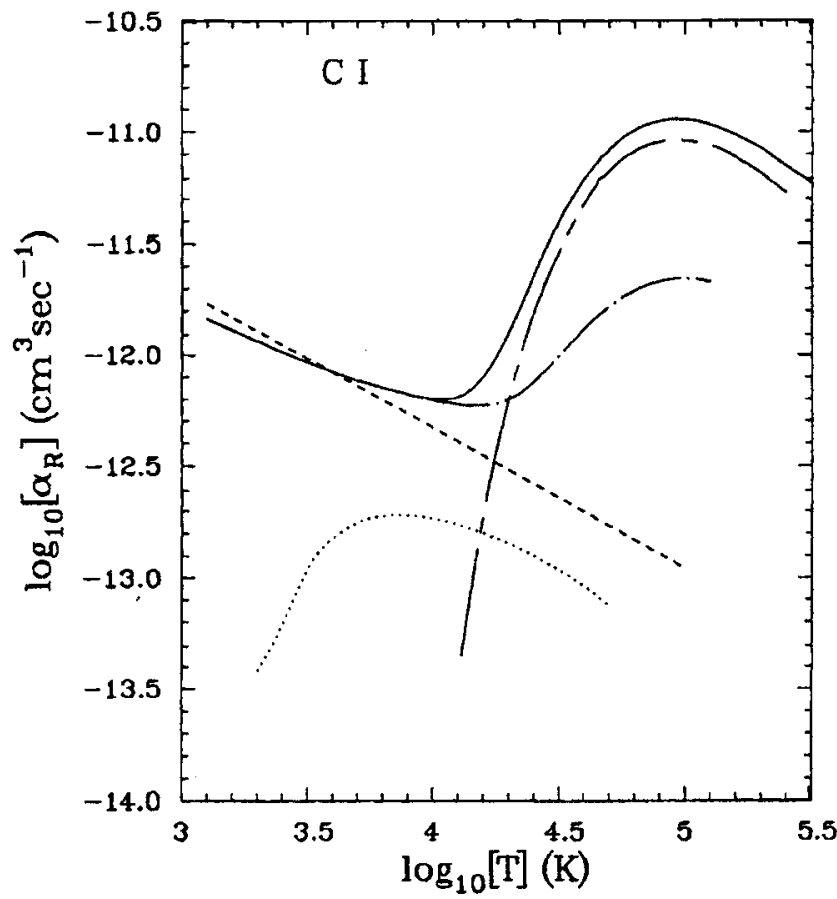

Fig. $5 a$

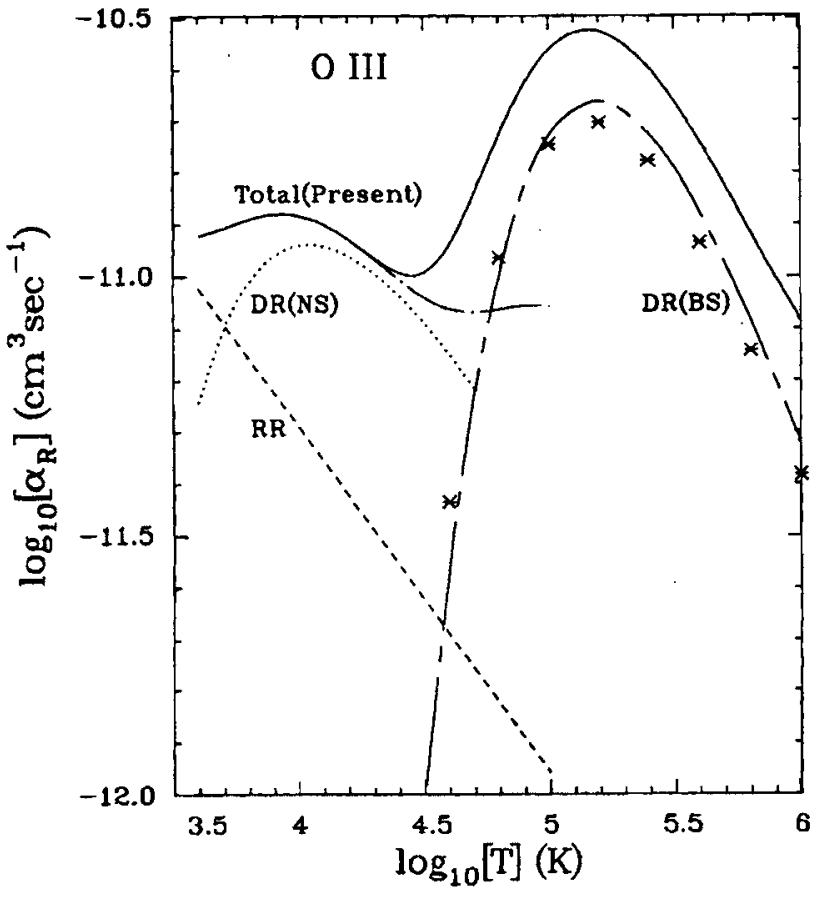

FIG. $5 c$

FYG. 5.-Recombination rate coefficients for $(a) \mathrm{C}_{1},(b) \mathrm{N} \mathrm{n}$, and (c) $\mathrm{O}$ in. In each figure the solid curve represents the present total $\alpha_{R}$; the dotted-chain curve represents contributions from states $n \leq 10$; the dashed-chain curve is the DR contribution for $n>10$ using the Bell \& Seaton theory (BS); the dashed curve represents BR rate coefficients by Aldrovandi \& Pequignot (1973); and the dotted curve is the DR rate coefficients by Nussbaumer \& Storey (NS, 1983). The asterisks in $(c)$ are the DR rate coefficients by Badnell $\&$ Pindzola (1989).

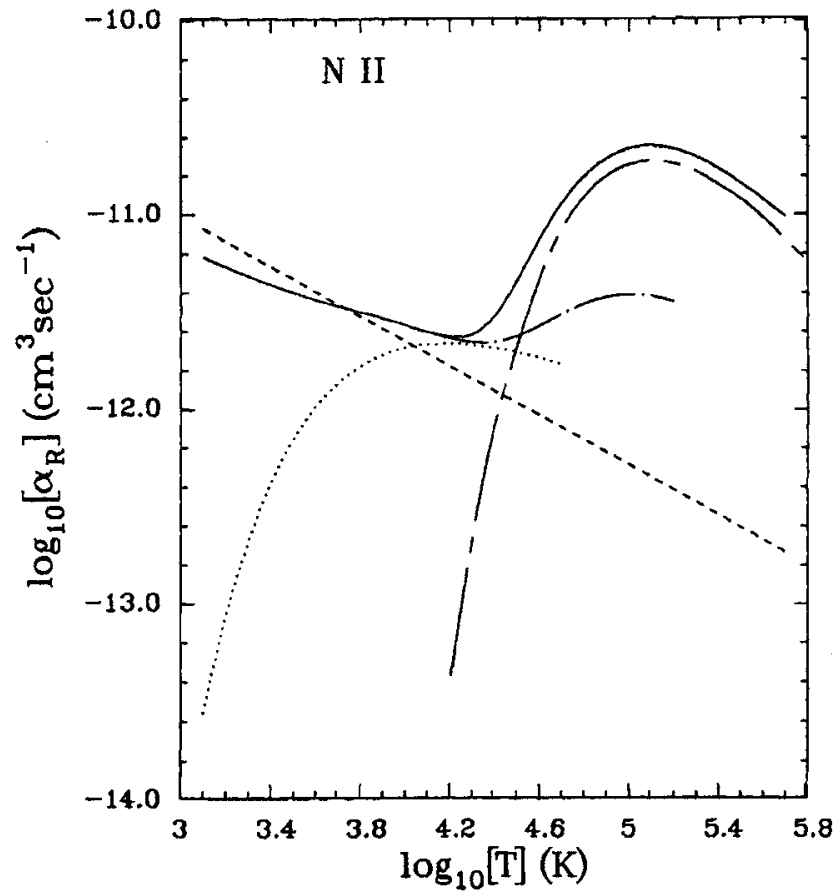

Fig. $5 b$

and compare these in detail with previously calculated $R R$ and DR rate coefficients. The present total rates are given by the solid curve, along with the contribution from the $n \leq 10$ states (broken curve with dots) using detailed photoionization cross sections and the Milne relation; the latter is seen as the continuation of the total curve from the point where the $n>10 \mathrm{DR}$ contribution (dashed chain curve) is included. The lowtemperature DR rate coefficients by Nussbaumer \& Storey (1983) are shown as the dotted curves and the purely RR rate coefficients by Aldrovandi \& Pequignot (1973) as the dashed curves. The RR values were computed using photoionization cross sections that did not include any resonance structure. The purely DR contribution to the present total rate coefficients, obtained using the Bell \& Seaton theory, lies below the high-temperature rise seen in all the total recombination rates; the difference between the total and the DR-only contribution may be viewed as the "background" contribution from the nonresonant region.

The present results for $\mathrm{O}$ II (Fig. $5 c$ ) are to be compared with the sum of three different and separate calculations for recombination: (i) the RR calculations of Aldrovandi \& Pequignot (1973), (ii) the low-temperature DR rate coefficients of Nussbaumer \& Storey (1983) and (iii) the high-temperature rate coefficients using the Burgess formula or the more recent work by Badnell \& Pindzola (1989). Although completely different methods have been employed, the present $O$ III results confirm the low-temperature rise in the rates due to the low-lying autoionizing resonances first discussed by Seaton \& Storey (1976) and later by Nussbaumer \& Storey (1983, hereafter NS). That the level of agreement is remarkable can be judged by the fact that the present results represent the use of detailed photoionization cross sections of 194 states of $\mathrm{O}$ III of the kind shown in Figure 1 in the calculations using equation (1), whereas the NS calculations employ a simpler technique of 


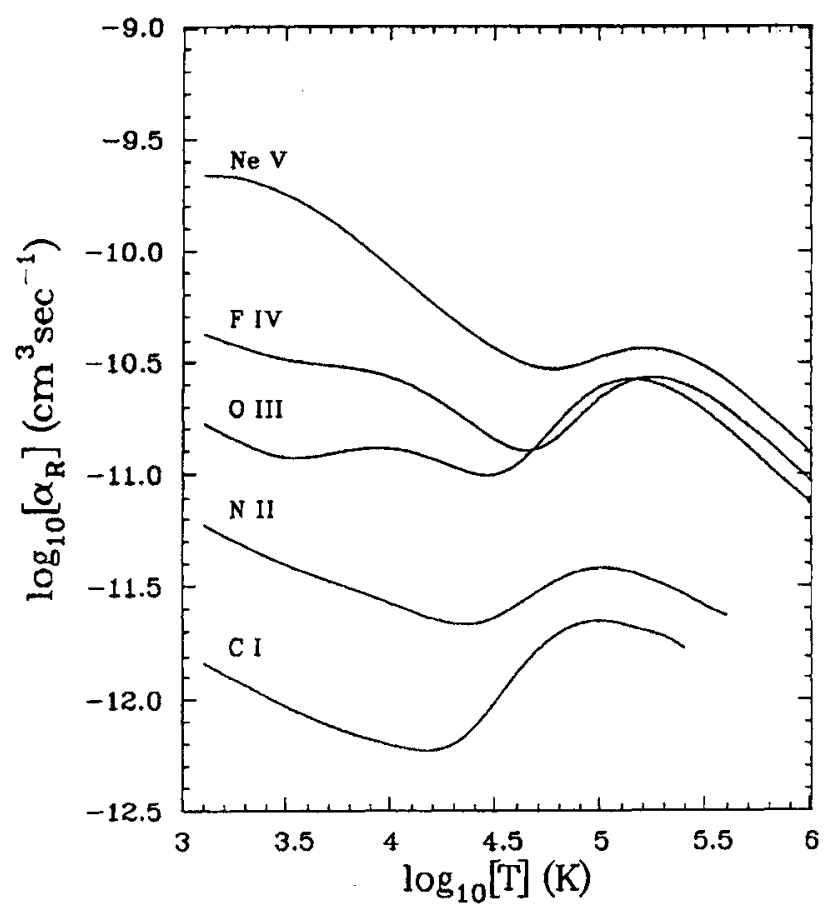

Fig. 6.-Total recombination rate coefficients, $\alpha_{R}$, for $C_{I}, N$ u, O II, F IV, and Ne v.

calculating the rate coefficient by treating autoionizing states to decay radiatively as bound states. The basic point, that low-lying autoionizing states with large autoionization rates may make a considerable contribution to DR at low temperatures, far in excess of the RR process, is shown to be the case for $\mathrm{O}$ III. However, the NS results are valid only in the low-temperature region. They recommend that for the total DR rate, their results should be augmented by the hightemperature contribution such as by the general formula by Burgess. It is seen from Figure $5 c$ that in the intermediate- and high-temperature region, the present results are significantly larger $(20 \%-30 \%)$ than the sum of the earlier RR plus the "low" temperature and the "high" temperature DR rates. In NP2, a comparison was mistakenly made at $\log T=4.7$ between the present total recombination rate coefficient and the sum of RR and DR (NS) values for $O$ III without including the high-temperature contribution. The rather confusing situation is further compounded by the fact that the different sets of contributions in previous works usually employ different atomic physics approximations and hence subject to different uncertainties. On the other hand, the present work includes not only the "background" photoionization cross section but all resonances, with detailed profiles for $n \leq 10$ and an accurate DR treatment of the ones with $n>10$, and hence yields a single, effective recombination rate.

The final results for the total $e+$ ion recombination rate coefficients, $\alpha_{R}$, are shown in Figure 6 for the five C-like ions $\mathrm{C}_{\mathrm{I}}, \mathrm{N}$ II, $\mathrm{O}$ III, F IV, and Ne V. Table 1 presents the numerical values of the rate coefficients tabulated at a wide temperature range for general astrophysical applications. The lowtemperature enhancement of the recombination rate coefficient, as in $\mathrm{O}$ IIl, is not a general phenomenon and is not present in $\mathrm{C} \mathrm{I}, \mathrm{N}$ Il, and $\mathrm{F}$ IV. It may be inferred that there are
TABLE 1

TOtal Reoombnatton Rate COEFicients"

\begin{tabular}{|c|c|c|c|c|c|}
\hline \multirow[b]{2}{*}{$t$} & \multicolumn{5}{|c|}{$\alpha_{R}$} \\
\hline & $C_{1}$ & $\mathrm{~N}$ II & $\mathrm{O} \mathrm{mI}$ & $\mathbf{F} \mathbf{~ v V}$ & $\mathrm{NeV}$ \\
\hline $3.0 .$. & $1.64(1)$ & $6.64(1)$ & $1.87(2)$ & $4.55(2)$ & $2.11(3)$ \\
\hline $3.1 \ldots$ & $1.46(1)$ & $5.93(1)$ & $1.68(2)$ & $4.21(2)$ & $2.19(3)$ \\
\hline $3.2 .$. & $1.30(1)$ & $5.31(1)$ & $1.50(2)$ & $3.89(2)$ & $2.18(3)$ \\
\hline $3.3 \ldots$ & $1.16(1)$ & $4.76(1)$ & $1.35(2)$ & $3.62(2)$ & $2.10(3)$ \\
\hline $3.4 \ldots \ldots \ldots$ & $1.03(1)$ & $4.30(1)$ & $1.24(2)$ & $3.41(2)$ & $1.96(3)$ \\
\hline $3.5 \ldots \ldots \ldots$ & 9.27 & $3.91(1)$ & $1.19(2)$ & $3.25(2)$ & $1.79(3)$ \\
\hline $3.6 \ldots$ & 8.39 & $3.60(1)$ & $1.19(2)$ & $3.15(2)$ & $1.59(3)$ \\
\hline $3.7 \ldots$ & 7.67 & $3.34(1)$ & $1.23(2)$ & $3.07(2)$ & $1.39(3)$ \\
\hline $3.8 \ldots$ & 7.10 & $3.11(1)$ & $1.28(2)$ & $2.99(2)$ & $1.19(3)$ \\
\hline $3.9 \ldots$ & 6.64 & $2.88(1)$ & $1.31(2)$ & $2.88(2)$ & $1.00(3)$ \\
\hline $4.0 \ldots$ & 6.27 & $2.66(1)$ & $1.30(2)$ & $2.71(2)$ & $8.40(2)$ \\
\hline $4.1 .$. & 6.00 & $2.46(1)$ & $1.25(2)$ & $2.48(2)$ & $6.99(2)$ \\
\hline $4.2 \ldots$ & 5.93 & $2.28(1)$ & $1.17(2)$ & $2.21(2)$ & $5.83(2)$ \\
\hline $4.3 \ldots \ldots \ldots$ & 6.32 & $2.17(1)$ & $1.08(2)$ & $1.93(2)$ & $4.09(2)$ \\
\hline $4.4 \ldots$ & 7.55 & $2.17(1)$ & $1.01(2)$ & $1.65(2)$ & $4.18(2)$ \\
\hline $4.5 \ldots$ & 9.88 & $2.32(1)$ & $9.98(1)$ & $1.42(2)$ & $3.64(2)$ \\
\hline $4.6 \ldots$ & $1.31(1)$ & $2.61(1)$ & $1.10(2)$ & $1.29(2)$ & $3.24(2)$ \\
\hline $4.7 \ldots$ & $1.67(1)$ & $3.01(1)$ & $1.35(2)$ & $1.30(2)$ & $3.01(2)$ \\
\hline $4.8 \ldots$ & $1.97(1)$ & $3.40(1)$ & $1.72(2)$ & $1.48(2)$ & $2.97(2)$ \\
\hline $4.9 \ldots \ldots \ldots$ & $2.17(1)$ & $3.69(1)$ & $2.13(2)$ & $1.82(2)$ & $3.10(2)$ \\
\hline $5.0 \ldots \ldots \ldots$ & $2.23(1)$ & $3.80(1)$ & $2.45(2)$ & $2.19(2)$ & $3.33(2)$ \\
\hline $5.1 \ldots$ & $2.16(1)$ & $3.73(1)$ & $2.63(2)$ & $2.51(2)$ & $3.54(2)$ \\
\hline $5.2 \ldots \ldots \ldots$ & $2.03(1)$ & $3.49(1)$ & $2.63(2)$ & $2.68(2)$ & $3.64(2)$ \\
\hline $5.3 \ldots \ldots \ldots$ & $1.91(1)$ & $3.20(1)$ & $2.47(2)$ & $2.68(2)$ & $3.58(2)$ \\
\hline $5.4 \ldots \ldots \ldots$ & $1.69(1)$ & $2.92(1)$ & $2.22(2)$ & $2.52(2)$ & $3.36(2)$ \\
\hline $5.5 \ldots \ldots \ldots$ & $1.67(1)$ & $2.57(1)$ & $1.91(2)$ & $2.27(2)$ & $3.01(2)$ \\
\hline $5.6 \ldots$ & & $2.43(1)$ & $1.60(2)$ & $1.96(2)$ & $2.62(2)$ \\
\hline $5.7 \ldots$ & $\ldots$ & ... & $1.32(2)$ & $1.65(2)$ & $2.20(2)$ \\
\hline & . & $\ldots$ & $1.07(2)$ & $1.38(2)$ & $1.82(2)$ \\
\hline & $\ldots$ & $\ldots$ & $8.91(1)$ & $1.13(2)$ & $1.51(2)$ \\
\hline $6.0 \ldots \ldots \ldots$ & $\ldots$ & $\ldots$ & $7.41(1)$ & $9.38(1)$ & $1.23(2)$ \\
\hline
\end{tabular}

$=\alpha_{R}$, in units of $10^{-13} \mathrm{~cm}^{3} \mathrm{~s}^{-1}$ at $t=\log _{10}[T(K)]$ for ions in the carbon sequence. The notation $a(b)$ means $a \times 10^{b}$.

no strongly autoionizing features in the near-threshold photoionization region for these ions. The level of detail in these calculations is indicated by the fact that 159 bound states of $\mathrm{C}$ I, 171 of $\mathrm{N} \mathrm{n}, 194$ of $\mathrm{O} \mathrm{m}, 231$ of F IV, and 256 of $\mathrm{Ne} v$ are included. In addition, the DR collision strengths have been calculated in the close coupling approximation including radiative decays from all dipole transitions from the excited states to the ground state of the core ions, and including coupling among the various autoionization channels.

As the present treatment considers resonance profiles in detail, it is expected to be more accurate than previous calculations using separate and simpler methods for radiative and dielectronic recombinations. However, two points might be made regarding the new results that concern radiative decays near the first ionization threshold and the relativistic effects. The first point is that the radiative decay of near-threshold resonances is in general small compared to decay into the continuum and is not considered for low-lying resonances $(v \leq 10.0)$; this may introduce some uncertainty. The use of the Milne relation based on detailed balance for autoionizing profiles analogous to the continuum cross sections, assumes that $A_{a} \gg A_{r}$, where $A_{a}, A_{r}$ are respectively the autoionization and radiative decay probabilities per unit time. Thus radiative decays to bound states of the $(e+i o n)$ system other than the initial photoionized state is not included. However, the approximation should be valid for most of the low-lying autoionization states, with the exception of the ones that are 
extremely narrow. This is evident by the fact that the present $\alpha_{R}(T)$ for $\mathrm{O}$ III in the low-temperature range agrees with and shows the same "bump" as the NS work which does take into account the radiative decays to other excited states. In general we expect rather small errors in the low-temperature region until we reach highly charged ions where the enhanced decay probability $\left(A_{r} \sim Z^{4}\right)$ of the low-lying autoionizing states will tend to make the present low-energy treatment increasingly uncertain. However, it should be ameliorated by the fact that we include, in the calculation of low-temperature $\alpha_{R}(T)$, the photoionization of a very large number of excited bound states and thus most radiative decays of autoionizing resonances should have been accounted for. In any event, for atoms and ions with $Z \leq 10$ we expect little uncertainty in the total recombination rates, but further work will be carried out before extending the present approach to heavier and more highly charged systerns. The second point is that while the relativistic effects are negligible on photoionization of these charged ions (Chen 1987), there may be contributions to DR process from transitions not allowed in the LS coupling. Work on recombination rates is in progress on other ions of the carbon sequence and other isoelectronic sequences using the present formulation as well as in Breit-Pauli approximation.

\section{CONCLUSION}

The new results from the Opacity Project are considerably at variance with presently available data for photoionization cross sections, in particular for the excited states of atoms and ions where the energy behavior is markedly nonhydrogenic. The new calculations have been carried out for the photoionization of a large number of bound states of each atomic or ionic system. As the autoionization effect is included in an ab initio manner in the close coupling formulation employed, total (electron + ion) recombination rate coefficients may be calculated with higher accuracy than hitherto possible. The new photoionization and recombination data are likely to be of importance in astrophysical models of photoionization regions in, for example, AGNs, quasars, and stellar atmospheres. While the new recombination rates will affect ionization balance in both coronal and nebular approximations, it is the photoionization data, displaying extensive resonance structures, that might considerably impact the modeling in the nebular case. It is expected to be a nontrivial task to incorporate the large amount of photoionization data into existing codes. Numerical approximations need to be developed for rapid convolutions over the radiation field particular to the given radiative source. Work is in progress along these lines using the Opacity Project data (G. J. Ferland, private communication).

Detailed consideration of the photoionization of excited states shows that the hydrogenic treatment or other simpler approximations for complex atoms and ions are inadequate. Further work is in progress on: (i) total recombination rate coefficients for a number of astrophysically important atomic systems, (ii) recombination rates for specific excited states important for the formation of recombination lines, and (iii) partial photoionization cross sections for non-LTE astrophysical models.

This work was supported in part by a grant from the National Science Foundation (AST-8996215). S. N. N. is the recipient of a Postdoctoral Research Fellowship awarded by the College of Mathematical and Physical Sciences at the Ohio State University. The computational work was carried out at the Ohio Supercomputer Center. We should like to thank Dr. Gary Ferland for discussions.
Aldrovandi, S. M. W., \& Pequignot, D. 1973, A\&A, 25, 137

Badnell, N. R., \& Pindzola, M. S. 1989, Phys. Rev. A, 39, 1690

Bell, R. H., \& Seaton, M. J. 1985, J. Phys. B, 18, 1589

Berrington, K. A., Burke, P. G., Butler, K., Seaton, M. J., Storey, P. J., Taylor, K. T. \& Yu. Y. 1987, J. Phys. B, 20, 6379

Chen, M. H. 1987, Phys. Rev. A, 35, 4586

Nahar, S. N., \& Pradhan, A. K. 1991, Phys. Rev. A, 44, 2935 (NP1)

—. 1992a, Phys. Rev. Lett., 68, 1488 (NP2)

1992b, Phys. Rev. A, in press (NP3)

\section{REFERENCES}

Nussbaumer, H., \& Storey, P. J. 1983, A\&A, 126, 75 (NS)

Osterbrock, D. E. 1989, Astrophysics of Gaseous Nebulae and Active Galactic Nuclei (Mill Valley, CA: University Science Books)

Pradhan, A. K. 1987, Phys. Scripta, 35, 840

Seaton, M. J. 1987, J. Phys. B, 20, 6363

Seaton, M. J., \& Storey, P. J. 1976, in Atomic Processes and Applications, ed. P. G. Burke \& B. L. Moiseiwitsch (North-Holland)

Yu, Y, \& Seaton, M. J. 1987, J. Phys. B, 20, 6409 